mind, since it would resolve many of the inconsistencies in different people's results.

Jaakkola: In their standard context, the low values of $\mathrm{M} / \mathrm{L}$ and $\Omega$, obtained recent1y in some we11-known investigations, imply an "open" Universe with hardly any influence of galaxy concentrations on the Hubble flow. The picture of steady recessional motion of galaxies resembles conspicuously the mechanistic world picture of 200 years ago and, therefore, it possesses in a certain sense a metaphysical content. One can even notice a feed-back to the ancient Ptolemaic idea of "perfect motion". The usual inference about a smooth redshift-distance relation as an argument in favour of the expansion hypotheses is incorrect. The case is actually the opposite - Hubble himself considered this result as a natural consequence of a photon interaction mechanism for redshifts and he may be taken as a fair authority as regards the Hubble relation! Taking into account the fundamental role of gravitation and the absurdity of dynamics without matter, a low value of $\Omega\left(\equiv \rho_{\text {obs }} / \rho_{\text {crit }}\right)$ should be considered as a conflict between theory ( $\rho_{\text {crit }}$ ) and observations ( $\left.\rho_{\text {obs }}\right)$.

\title{
STABILIZATION OF SYSTEMS OF GALAXIES BY SUBCLUSTERING
}

\section{M. Ozernoy and M. Reinhardt}

Subclustering might help to solve the virial theorem paradox for systems of galaxies by hiding a major part of the potential energy in gravitationally bound subsystems. We have shown (Ozernoy and Reinhardt 1976, Astr. Astrophys., 52, 31) that even in groups of galaxies there is mass segregation, in the sense that bright group members tend to be concentrated towards the centre. Recently Wesson and Lermann (1977, Astrophys. Sp. Sci., 46, 327), realizing the importance of subclustering, proposed a quantitative method for estimating its effect on the stability of systems of galaxies. However, their assumption about the frequency of subsystems of multiplicity $n$ is not in accord with Holmberg's (1962) result. The mean frequency of galaxies in pairs is 0.37 for the Turner and Gott groups (1976) and 0.23 for the de Vauceulours groups (1976), in good agreement with the value of 0.25 required by Holmberg's distribution. Assuming Holmberg's frequency of gravitationally bound subsystems and that they are homogeneously distributed throughout the system, we have for the ratio of the total potential energy of a system of $\mathrm{N}$ equal masses $\Omega$ to the potential energy calculated in the usual way neglecting subclustering $\Omega_{\mathrm{S}}, \Omega / \Omega_{\mathrm{S}} \approx 1+\left(\mathrm{R}_{\mathrm{c}}\right) /\left(<\mathrm{r}_{2}>\mathrm{N}\right)$, if the velocity dispersion $\left\langle\sigma_{r}{ }^{2}(n)\right\rangle=$ constant. Here $R_{c}$ is the effective radius of the system and $\left\langle r_{2}\right\rangle$ the mean distance of binaries. The assumption $\sigma_{r}{ }^{2}(n)=$ const is reasonable for $n \leqslant 7$, when Holmberg's distribution holds, since $\sigma_{\mathrm{r}}{ }^{2}(2)=203 \mathrm{~km} \mathrm{~s}^{-1}$ according to Karachentsev (1974), and increases to only $\simeq 1000 \mathrm{~km} \mathrm{~s}^{-1}$ for rich clusters. Since Karachentsev's data give an $\left\langle\mathrm{r}_{2}\right\rangle=33 \mathrm{kpc}$ for $\mathrm{H}_{\mathrm{O}}=55 \mathrm{~km} \mathrm{~s}^{-1} \mathrm{Mpc}^{-1}$, we have $\Omega / \Omega_{S} \approx 4$ for groups of galaxies with $R_{c} \approx 1 \mathrm{Mpc}$ and $N=10$. Thus it seems that subclustering cannot remove the mass discrepancy for $\mathrm{rich}$ clusters and for groups only in moderate cases. 
However, so far we have considered only systems of galaxies of equal mass, which is clearly unrealistic. Particularly in rich clusters, we often find two very bright galaxies in the central region, which might form a physical pair. For example, if NGC 4874/4889 in the Coma cluster are gravitationally bound, the binding energy of this pair alone would exceed $\Omega_{\mathrm{s}}$ for the whole cluster, derived by the usual method, by a factor of 11.5 for $R_{c} \approx 2 \mathrm{Mpc}$ and $\mathrm{N} \approx 800$. Thus this single pair could stabilize the whole Coma cluster. It is interesting that irregular clusters of galaxies, which on the average show a particularly high mass discrepancy, have also a high number of multiple subsystems.

For groups we give as an example Nos 48 and 49 in de Vaucouleur's catalogue (1976). The ratio of the binding energy of the pairs NGC $3504 / 3512$ and NGC $3607 / 3608$ to $\Omega_{s}$ is $\Omega_{p} / \Omega_{s}=15.4$ and 10.4 , respectively, as compared to the observed mass discrepancy $\mathrm{M}_{\mathrm{VT}} / \mathrm{M}=9.1$ and 10.9 (Rood et a1. 1970). From this we draw the tentative conclusion that subclustering, especially binary galaxies, must be taken into account in the correct calculation of the potential energy of systems of galaxies and that this might remove the virial mass paradox, where it still seems to exist. This would also be important for the determination of the cosmological density parameter $\Omega$.

\section{DISCUSSION}

Tumer: You suggest the substitution of a hierarchical mass model for the usual continuous one in the evaluation of the potential energy. Neither approximation is necessary, of course; the potential energy may be evaluated directly from the observed positions of the individual galaxies. This is the standard procedure for small groups and has been used also on rich clusters. For the Coma cluster such direct evaluation of the potential energy agrees fairly well with that derived from the continuous mass model.

Reinhardt: Calculating the potential energy of a given system of galaxies from the observed positions, you do not know, because of projection effects, whether some galaxies form gravitationally bound subsystems or not. On the other hand there is a convincing statistical evidence of the existence of such substructures, as we have pointed out. Therefore, it is necessary to take into account the binding energy of subsystems in the calculation of the potential energy. Neglecting subclustering will in general give a wrong result.

Tumer: I agree that the two bright galaxies at the centre of Coma may well be a binary; I do not agree that this binary dominates the cluster potential energy.

Reinhardt: We are happy that you agree with us in that the two bright galaxies in Coma might form a bound pair. However, in this case it follows directly from the data that its binding energy greatly exceeds that of the whole cluster. There is no way out of it. 
Peebles: If you want to assume the mass is mainly in the two brightest galaxies near the centre of the Coma cluster then the potential varies as $\mathrm{r}^{-2}$, and to match the observed counts of galaxies as a function of distance from the centre of the cluster, number density $N(r) \propto r^{-\infty}$, you must assume the velocity dispersion varies as $\mathrm{v}^{2} \propto \mathrm{r}^{-1}$, which seems not to be observed.

Reinhardt: I think you have misunderstood me. We assume that in the central pair of galaxies there is concentrated only a small part of the total mass of the system, but a large part of the binding energy. Of course, this is not the same and our assumption does not require any change of the velocity distribution.

\section{MORPHOLOGICAL INVESTIGATION OF PAIRS CONTAINING MARKARIAN GALAXIES* \\ C. Casini and J. Heidmann}

We have obtained large scale photographs or electronographs for 40 pairs containing Markarian galaxies: 6 Markarian-Markarian pairs and 34 Markarian-normal pairs, most of which are physical close pairs with two similar components (here "normal" means with no UV excess).

We have studied the distribution of types for the Markarian and for the normal components; the most frequent type is spiral, with more ordinary spirals than barred ones; next are compact for the Markarian and elliptical for the normal galaxies; the only one irregular is of clumpy type.

A comparison was made between isolated Markarian galaxies and those in pairs; for both, spirals are dominant with ordinary ones more frequent than barred ones. There are more compacts, fewer irregulars and much fewer lenticulars among pairs of Markarian galaxies than among isolated ones.

Morphological peculiarities have been investigated. In the Markarian-normal pairs, the Markarian is more often peculiar; thus peculiarity is associated with UV excess; there is no tendency for peculiar Markarian galaxies to pair with peculiar normal galaxies; peculiar Markarians have more often a diffuse spectrum but it cannot be said that morphological peculiarities are located at the same positions as the UV excess.

Six noteworthy peculiar galaxies are described which contain double strings of condensations, double or triple nuclei, clumpy structure or whorl shape.

*Submitted to Astr. Astrophys. Suppl. Series. 